\title{
False Belief Understanding and Moral Judgment in Young Children
}

Karlena D. Ochoa ${ }^{1}$, Joseph F. Rodini ${ }^{1}$, and Louis J. Moses ${ }^{2}$

${ }^{1}$ University of Oregon, ${ }^{2}$ Victoria University of Wellington

\section{Author Note}

The authors confirm that there are no known conflicts of interest.

Correspondence concerning this article should be addressed to Karlena D. Ochoa, Department of Psychology, University of Oregon, 1227 University of Oregon, Eugene, OR 97403

E-mail:kochoa@uoregon.edu 


\begin{abstract}
Although the influence of intent understanding on children's moral development has been long studied, little research has examined the influence of belief understanding on that development. In two studies we presented children with morally-relevant belief vignettes to examine the extent to which they incorporate both intent and belief information in their moral judgments. In Study $1(N=64), 5$-year-old false belief $(\mathrm{FB})$ passers rated agents with false beliefs as more positively intentioned in good intent trials (even though the outcome was bad) than in bad intent trials (even though the outcome was good). In contrast, 4-year-old FB passers were generally unable to integrate their belief understanding with their moral evaluations, performing no better on intention questions than FB failers. Neither age group significantly differentiated rewards and punishments as a function of intent when a false belief was involved. In Study $2(N=109$ children, $N=42$ adults), we found that by simplifying our study design and reducing the task demands, 4-year-old FB passers' were able to make appropriate intent judgments. Yet, as in Study 1, all children had difficulty assigning punishment/rewards based on intent. For both moral intentions and moral consequences, 4- and 5-year-old false belief passers' moral judgments differed from those of adults in several respects, indicating that moral reasoning develops substantially beyond the preschool years.
\end{abstract}

Keywords: Moral Judgments, Theory of Mind, Development, Social Cognition 
False Belief Understanding and Moral Judgment in Young Children

Sometime during the preschool years, before formal schooling, children begin to display understanding of their own and others' mental states such as desires, beliefs, intentions, and emotions. This Theory of Mind (ToM), allows them to understand, explain, and predict behavior (Premack \& Woodruff, 1978), and is critical for other domains of social functioning (Astington, 2003; Baron-Cohen et al., 1985; Leslie, 1988; Wellman, 2020). One such domain is that of moral judgment (Baird \& Astington, 2005; Killen et al., 2011; Lane et al., 2010; Lagattuta \& Weller, 2014; Smetana, et al., 2012; Wainryb, \& Ford, 1998). The current paper addresses the interplay between ToM and moral judgment in young children.

Understanding intentions is one aspect of ToM that is central in moral judgment. In our everyday ethical reasoning, as well as in our legal codes, the intentions that underlie actions are critical in assessing the moral status of actors and whether or not their actions are deserving of reward or punishment. Yet there is a long history of research going back at least to Piaget (1932) indicating that young children often rely more on outcomes than intentions when making moral judgments (Cushman et al., 2013; Killen et al., 2011; Zelazo et al., 1996). Nonetheless, in simple scenarios in which the outcome is unknown or otherwise held constant, even children as young as three can make appropriate use of information about intent in making moral judgments (Smetana, 2006).

Understanding others' beliefs, including false beliefs, is also important for making moral judgments. In contrast to intentions, however, there has been very little research assessing how and when children integrate belief information into their moral judgments. Although some earlier work indirectly examined belief and knowledge understanding in relation to moral development (Wimmer et al., 1984; Yuill \& Perner, 1988), it is only 
recently that a systematic analysis of the relation has been undertaken. Killen et al. (2011) assessed 3- to 8-year-old children's understanding in a moral transgression task embedded within a false belief story. In this story, a well-intentioned boy accidentally causes a negative outcome because he acts on the basis of a false belief about a container's contents.

Specifically, as the boy was helping a teacher clear tables, he threw out a paper bag which, unbeknownst to him, contained another child's cupcake. Children then responded to a range of questions including those assessing their false belief understanding (i.e., what did the boy believe was in the bag?), their moral appraisal of the actor's intention (i.e., whether the boy thought he was doing something all right or not all right), and, in a second study, whether punishment was warranted (i.e., whether the boy should get in trouble). Killen et al. found that children without false belief understanding were more likely to attribute negative intentions and to assign punishment to the accidental transgressor than children with false belief understanding. Moreover, it was not until children were 7 or 8 that they attributed positive intentions to the boy at high levels.

This research establishes a link between belief understanding and moral judgment in childhood, but leaves many questions unanswered. First, Killen et al. (2011) administered only one morally-relevant belief story with only one combination of agent's belief (false) and intention (good). It remains to be seen whether there are developmental changes across other combinations of belief (true vs. false) and intention (good vs. bad), and whether belief and intention interact in some way. Children may have had similar difficulty even in a true belief context and may have responded differently had the intentions of the actor been negative.

Second, in the Killen et al. (2011) study, to perform well children were required to make assumptions about the agent's intention (i.e., that the boy cleaning tables would not have thrown out the cupcake had he known it was in the bag). Hence, the younger children may 
have performed poorly either because they lacked false belief understanding or because they did not hold a default assumption of benign intent. They might show greater ability to incorporate belief understanding with moral evaluations if agents' intentions are made more explicit.

Third, when children initially attain false belief understanding, they may still have difficulty applying that knowledge to moral judgments. There may be a lag such that their ability to integrate their newly acquired belief understanding with their moral understanding is delayed. Interestingly, a similar lag has been found with respect to integrating belief information with emotion understanding. De Rosnay et al. (2004) told children stories in which, for example, a character mistakenly believed that a container held a preferred food when in actuality it contained a disliked food. Although 4- and 5-year-olds understood the character's mistaken belief, they nonetheless incorrectly predicted that the character would feel sad upon seeing the container. It was not until age 6 that children made correct emotion predictions (see also, Wellman \& Liu, 2004). This finding, that children cannot immediately use belief information to inform judgments about another mental state (emotion), demonstrates difficulty in integrating concepts within the ToM domain. It remains to be seen whether similar difficulty, and a similar developmental lag, would be found in integrating belief reasoning with understanding in a different domain, that of moral judgment. Preliminary evidence for such a lag comes from the Killen et al. (2011) study. They found that while 7-year-olds rated the accidental transgressor as having positive intentions, 5-year-olds (most of whom correctly answered the false belief question) gave a neutral rating of the agent's intention. Whether that finding is replicable for positive intentions and extendable to negative intentions is not known. 
Finally, a lag may also be present between children's appreciation of moral intentions and their accurate assignment of consequences to actors. For example, Cushman et al. (2013) found that around age 5 children first incorporate intent information into judgments of wrongness and only later, between 6-8 years, into intent-based punishment judgments. They argue that this pattern is not driven by changes in relation to ToM but instead represents the reorganization of concepts within the moral domain itself, specifically in how intent relates to wrongness and punishment. While Killen et al. (2011) questioned children regarding both moral intentions and moral consequences, their data are not broken down in such a way as to clearly determine whether the latter were more difficult to appreciate than the former.

The current research was designed to address these issues. In two studies we manipulated agents' intentions and beliefs in morally-relevant vignettes to examine preschoolers' ability to integrate belief understanding with moral judgment. In both studies we assessed children's understanding of beliefs, moral intentions, and moral consequences across the age range (4-5 years) in which children are acquiring false belief understanding. Doing so allowed us to determine whether developmental lags are present for integrating information across the domains of ToM and moral judgment, whether they are present for reasoning about both positive and negative moral intentions, and whether they are present for reasoning about both true and false beliefs.

\section{Study 1}

In Study 1, we manipulated agents' intentions (positive or negative) and beliefs (true or false) in vignettes conceptually comparable to those used in Killen et al. (2011). Each vignette featured an agent intending to deliver a benefit (positive intent) or a harm (negative intent) to another character. When the agent held a true belief, the harm or benefit was successfully delivered whereas when the agent held a false belief the opposite outcome 
occurred (e.g., delivering benefit when harm was intended and vice versa). Children were then questioned about agents' beliefs and intentions, and asked to assign reward or punishment. For comparison we also assessed children's false belief understanding in a standard non-moral context, and their moral understanding in a standard moral judgment task.

For true belief trials we hypothesized that children would rate agents in the good intent condition as morally superior to those in the bad intent condition, and would appropriately recommend reward or punishment. In contrast, we hypothesized that the intersection of intent and false belief would lead to differential response patterns across the sample concordant with the attainment of false belief understanding. We expected that children without false belief understanding would perform poorly, often responding in terms of outcome when assessing intent and assigning reward or punishment. For children with false belief understanding, we had two contrasting hypotheses. If children immediately integrate false belief understanding they would use this information to correctly infer agents' intentions. In contrast, if there is an integration delay, the younger children might perform poorly, often defaulting to outcome-based responding. Finally, based on prior research (Cushman et al., 2013), we hypothesized a further developmental lag between appropriately assigning intent and appropriately assigning consequences.

\section{Method}

\section{Participants}

Sixty-four children participated, 32 4-year-olds (13 girls; $M$ age $=53.56$ months, $S D$ $=4.00)$ and 325 -year-olds $(15$ girls; $M$ age $=66.91, S D=3.54)$. An additional 11 children were tested but excluded from analyses due to experimenter error (2), refusal to cooperate/inattention (4), and failure to provide sufficient answers for the morally-relevant belief vignettes (5). 
Children were typically developing and were recruited from a participant database at a large research university in the Western United States. The sample was representative of the population from which it was drawn. Forty-nine children were White, 6 were multiracial, 5 were LatinX, 2 were Native American/Native Alaskan, 1 child was African-American, and 1 child was Asian/Pacific Islander. Parents were provided compensation in the form of $\$ 10$, and children received a small toy. The research protocols for this and the following study were approved by the XXX Institutional Review Board.

\section{Procedure}

Children were tested individually in a single 45 -min videotaped session. The study was a part of a larger project examining theory of mind, executive functioning, and moral development. Here we present findings on ToM and moral development. Children received tasks in two counterbalanced testing blocks: a morally-relevant belief vignette block, and a standard moral transgression and false belief block. In the first block, vignettes were presented in a counterbalanced order, as were the tasks in the second block.

\section{Measures}

Morally-relevant belief vignettes. Children in each age group were randomly assigned to one of two conditions: good intent $(\mathrm{n}=32 ; 13$ girls; $\mathrm{M}$ age $=60.34$ months, $\mathrm{SD}=8.24)$ or bad intent $(\mathrm{n}=32 ; 15$ girls; $\mathrm{M}$ age $=60.13$ months, $\mathrm{SD}=7.24)$. Within conditions, belief (true or false) was manipulated across four vignettes (see Table 1). The vignettes all featured two characters, one of whom (the "agent") discovered a pleasant object in an opaque container and an unpleasant object in a different opaque container. The agent then decided to share one of these containers with another character (the "recipient") after the objects switched containers. The sharing of objects in these stories served as instances of harming or helping behavior. Two of the stories involved true belief (TB) and two involved false belief (FB) on 
the part of the agent (i.e., for two stories the agent knew the contents of the container and for two stories the agent had a mistaken belief about the contents of the container). The order of story presentation was counterbalanced across children and fully decoupled with respect to belief and intent (e.g., the kitten and skunk story served as a bad intent, TB story for some children and a good intent, FB story for other children). A depiction of the experimental manipulations and a vignette script can be found in supplemental material on the Open Science Framework (OSF) at https://osf.io/3p5m9/.

As an example, in the kitten and skunk story, Bobby (the agent) discovered a bucket containing a kitten and a bin containing a skunk. After returning the animals to their containers, he explicitly stated his intent to make a second, offstage character, Jacob (the recipient), either happy or upset via sharing the container with the kitten or skunk, respectively (in contrast to the Killen et al. (2011) study in which the intention needed to be assumed). The agent then left the scene, at which point the belief manipulation took place, with the agent either immediately returning to witness the animals switch location (TB trials) or returning only after the animals switched locations (FB trials). At this point, the recipient entered the scene, prompting the agent to offer either what he thought was a "good" container (the one containing the kitten) or what he thought was a "bad" container (the one containing the skunk) to the recipient. The other stories also featured positively and negatively valenced items: a butterfly and a spider, a cupcake and a moldy tomato, and a cookie and a rotten apple, respectively. In stories with valenced animals, the animals switched positions themselves, while in stories with valenced food items, a new animal appeared from offstage to switch the objects and left before the recipient arrived (a pet parrot and a field mouse, respectively). 
Children were asked three comprehension questions over the course of the story: two of these concerned whether each of the items would make the recipient feel good or bad (e.g., “Do kittens make Jacob feel good or bad?") and one concerned whether the agent was present or absent for the switch (e.g., "Was Bobby there to see the animals switch?"). Three trials in which children failed one or more of these comprehension questions were excluded. Also excluded were trials in which children (5) failed to pass the reality check question (see below).

After each vignette, children responded to seven questions in a fixed order: (1) intention evaluation ("When [AGENT] handed [RECIPIENT] the container, was [AGENT] trying to be a good boy, bad boy, or just okay?" (2) agent belief ("What does [AGENT] think is in the container?"); (3) reality check ("What is really in the container?"); (4) agent evaluation (“Do you think [AGENT] is being mean, nice, or just okay?”); (5) consequence evaluation ("Should [AGENT] get in trouble, like a timeout, a treat, like a trip to the zoo, or nothing? If [AGENT] gets nothing, he will not get a timeout or a trip.”); (6) friend evaluation (“Do you want to be friends with [AGENT]?"); and (7) recipient emotion attribution ("How will [RECIPIENT] feel when he opens the container?"). The consequence assignment question featured depictions of the three consequences to which the child could point. Children were credited with FB understanding only if they correctly answered the agent belief and reality check question.

Scoring for these measures fell into two schemes: Questions 2 and 3 (correct or incorrect); questions 1, 4, 5, and 7 (a continuous, three-point scale based on answer valence, with negatively-valenced answers coded -1 , neutral answers coded 0 , and positively-valenced answers coded 1). The intent evaluation and agent evaluation questions were significantly correlated in both the TB $(r(64)=.87, \mathrm{p}<.001)$ and FB conditions $(r(64)=.57, p<.001)$, 
and produced very similar results. For brevity, we combined them into a single item for analysis (the agent intention composite). The friend evaluation question seemed to be strongly affected by a yes response bias (many children wanted to be friends with all agents), and therefore is not analyzed further here, but data can be found in the supplemental material.

Standard moral transgression task. The moral transgression task consisted of a single, prototypic moral transgression story often used in the literature (see Smetana, 2006) that involved a character pushing someone off a swing. Children were presented with pictures of a swing set and two characters who matched their own gender. They were told a story in which one character pushes another off the swing, causing the second character to fall and hurt his/her knee. They then responded to a subset of questions from the morally-relevant belief vignettes: agent evaluation, consequence evaluation, friend evaluation, and recipient emotion attribution.

Standard false belief task. The standard FB task (adapted from Leslie et al., 2005) had a broadly similar structure to the moral vignettes and featured a boy (Jamie), two doghouses, and two dogs - one with spots and one without. After discovering the dogs, the boy was described as wanting to give a bone to the dog with spots. The boy then left to get the bone and, while he was away, the dogs switched places. The boy then returned with the bone and approached the doghouse in which the spotted dog was originally located. Children were then asked which dog the boy thinks is in the doghouse and which dog is really in it.

\section{Results}

\section{Standard Moral and False Belief Tasks}

In replication of previous research, children recognized that the agent in the prototypical moral transgression task was meaner than neutral ( "just okay"), $(M=-0.91, S D$ $=.43$ ), and deserved to be punished more harshly than the neutral (no punishment or reward), 
$M=-0.66, S D=0.62), t s(63)=-17.02$ and -8.43 respectively, $p s<.001$. Four and 5 -year-olds did not differ significantly for either the evaluation or consequence ratings. Further, almost all children recognised that the victim would feel sad $(M=-0.93, S D=0.37)$.

On the standard FB task, there was a marginally significant age effect with 5-year-olds somewhat more likely to exhibit FB understanding than 4-year-olds, $\chi^{2}(1, N=$ $59)=3.37, p=.07, \phi=.24 .4$-year-olds (62\% correct) did not differ from chance, $\chi^{2}(1, N=$ $29)=1.69, n s$, whereas 5-year-olds did (83\%), $\chi^{2}(1, N=30)=13.33, p<.001$.

\section{Comparison of Morally-Relevant Vignettes with Standard Tasks}

To assess the comparability of our moral vignettes and the standard moral transgression task, we compared responses across tasks to the agent intention and consequence questions from the half of the sample $(N=31)$ that were given the standard moral transgression task and the analogous TB, bad intent vignettes. Asthere was only one standard task, we compared it to only the first of the TB, bad intent vignettes that children received. Children rated the agent as meaner in the standard story $(M=-0.94, S D=0.35)$ than in the morally-relevant belief story $(M=-0.59, S D=0.76), t(30)=-2.99, p=.006$, and as more deserving of punishment in the standard story $(M=-0.72, S D=0.58)$ than in the morally-relevant belief vignette $(M=-0.10, S D=0.87), t(30)=3.45, p=.002$.

In a similar analysis, children's performance on the standard FB task (73\% correct) did not differ significantly from their performance on the belief question on the first morally-relevant FB vignette to which they responded (76\% correct), McNemar's test. Moreover, children's responses to the belief questions on these tasks were highly correlated, $\phi=.65, p<.001$.

\section{Morally-Relevant Belief Vignettes}


No main effects of gender, order (block one vs. block two), or story (e.g., kitten/skunk vs. others) were found for any morally-relevant belief vignette test items ( $p$ s $>.15)$, and so these factors were collapsed in subsequent analyses. All children in these analyses answered the reality check question correctly for at least one TB story and one FB story. Five children were excluded for failing all reality check questions.

We used the recipient emotion prediction question as a comprehension check. Consistent with earlier work with standard moral transgression tasks (Smetana, 2016), children appropriately judged that recipients in $\operatorname{good}$ intent TB $\operatorname{trials}(M=.92, S D=.26)$ would feel happy and that those in bad intent TB trials would feel sad $(M=-1, S D=0)$. Similarly, children judged that recipients would feel sad in FB good intent trials $(M=-.88$, $S D=.40)$ and happy in FB bad intent trials $(M=.81, S D=.40)$. Importantly, these results for the last question asked following each vignette suggest that children were not seriously hampered by memory demands across the task.

Agent Belief. A 2 (belief: true vs. false) x 2 (intent: good vs. bad) x 2 (age: 4- vs. 5-year-olds) mixed ANOVA, with repeated measures on the first factor, was conducted on responses to the agent belief question. A main effect of intent revealed children ascribing the appropriate belief more often to the agent in the good intent condition $(M=0.85, S D=0.22)$ than in the bad intent condition $(M=0.67, S D=0.28), F(1,60)=8.33, p<.01, \eta_{p}{ }^{2}=.12$. Also present was an interaction between belief and age, $F(1,60)=4.66, p=.03, \eta_{p}^{2}=.07$. As expected, while 4-year-olds $(M=0.86, S D=0.32)$ and 5-year-olds $(M=0.80, S D=0.40)$ did not differ in their ability to ascribe appropriate beliefs to the agent in TB trials, $t(60)=0.72, p$ $>.15$, 4-year-olds $(M=0.56, S D=0.49)$ were less likely than 5-year-olds $(M=0.83, S D=$ 0.37 ) to ascribe appropriate beliefs to the agent in FB trials, $t(60)=2.46, p=.02$. Further, 
while 4-year-olds were only above chance on TB trials, 5-year-olds were above chance for both TB and FB trials $(p \mathrm{~s}<.001)$.

The sample was then divided into three groups as a function of belief understanding: False belief failers -- children who failed one or both of the belief questions on the morally-relevant FB vignettes $(N=21 ; 7$ girls; $M$ age $=56.38$ months, $S D=6.30)$; 4-year-old passers -- children of that age who passed both questions $(N=17 ; 8$ girls; $M$ age $=53.94$ months, $S D=4.18$ ); and 5-year-old passers -- children of that age who passed both belief questions $(N=26 ; 13$ girls; $M$ age $=67.46$ months, $S D=3.56)$. This grouping factor best allows for the assessment of whether FB understanding is immediately coupled with advances in moral evaluation or whether there is a developmental lag in integrating these forms of reasoning. For the remaining analyses, we present results separately for TB and FB trials, with TB being reported first as a baseline from which to evaluate moral judgment in FB conditions.

Agent intent composite. Figure 1 depicts children's performance on the agent intent composite. For TB trials, a 3 (FB group) x 2 (intent) factorial ANOVA showed a main effect of intent, $F(1,58)=189.85, p<.001, \eta_{p}^{2}=.77$. Children appropriately rated agents in the good intent condition $(M=.67, S D=.50)$ as better intentioned than those in the bad intent condition $(M=-.81, S D=.34)$. The main effect of FB group and the interaction were not significant. For FB trials, a main effect of intent again emerged, with children in the good intent condition $(M=.37, S D=.60)$ appropriately rating agents as better intentioned than those in the bad intent condition $(M=-.03, S D=.68), F(1,58)=6.99, p=.01, \eta_{p}{ }^{2}=.11$. However, this main effect was qualified by a FB group by intent interaction, $F(2,58)=8.65$, $p<.001, \eta_{p}{ }^{2}=.23$. Follow up simple effects comparisons (Tukey adjusted) of intent for each of the three FB groups revealed that two groups, FB failers and 4-year-old passers, did not 
show a distinction for intention ratings between the good intent and bad intent conditions (both $p \mathrm{~s}=1.00$ ). In contrast, 5-year-old passers appropriately rated agents in the good intent condition $(M=.63, S D=.43)$ as significantly better intentioned than those in the bad intent condition $(M=-.48, S D=.66), t(58)=-4.88, p<.001$.

Agent consequence. Figure 2 depicts children's performance on the agent consequence measure. For TB trials, a 3 (group) x 2 (intent) factorial ANOVA revealed a main effect of intent. Children appropriately rated agents in bad intent conditions $(M=-.39$, $S D=.67)$ as deserving more punishment than those in good intent conditions $(M=.89, S D=$ $.28), F(1,58)=92.41, p<.001, \eta_{p}^{2}=.62$.

In contrast, in the FB condition there were no significant effects. Of all cell means, only FB failers' rating of agent consequence in the bad intent condition differed from chance, $(M=.54, S D=.69), t(58)=2.44, p<.05$, and was actually in the incorrect direction with many children stating that the bad-intentioned agent deserved a reward, underscoring FB failers' difficulty with this question. In general, while children were able to assign appropriate consequences in a TB context, doing so in a FB context was challenging for all groups.

We conducted a follow-up analysis, in the FB condition, recoding children's responses as either correct or incorrect. Specifically, in the bad intent condition, assigning trouble is a clear correct response but assigning nothing could also be considered reasonable as there is no negative outcome. Assigning a treat in this condition is clearly incorrect. Conversely, in the good intent condition, assigning a treat is a clear correct response but assigning nothing could also be considered reasonable as there is no positive outcome. Assigning trouble in this condition is clearly incorrect. Children thus received a score from 0-2 based on their correct/incorrect responses across the two trials coded as just described. A 
group by intent ANOVA revealed a main effect of intent, such that children made more correct consequence judgments in the good intent condition $(M=1.41, S D=.80)$ compared to the bad intent condition $(M=.78, S D=.91), F(1,58)=8.14, p=.006, \eta_{p}^{2}=.12$. As in the preceding analysis, however, there was no significant effect of groups status on consequence judgments. FB failers (43\% correct), 4-year-old passers (29\% correct), and 5-year-old passers (58\%) all had difficulty assigning consequences in the FB context, with no group performing better than chance, $t \mathrm{~s}(20,16,25)=-1.79,-1.96,1.31, p \mathrm{~s}>.09$.

\section{Discussion}

By limiting the age groups in our sample to the narrow window during which false belief understanding is acquired, we found intriguing interactions between ToM and moral judgments. The morally-relevant belief vignettes served as dual assessments of false belief understanding and moral reasoning, and elicited responses from a sample of 4-and 5-year-olds demonstrating developmental changes in moral reasoning across the acquisition of false belief understanding.

We first evaluated whether our moral tasks were in relevant respects comparable in difficulty to a standard false belief and a standard moral transgression task. Performance on false belief questions in the moral vignettes was no more difficult than that on the standard false belief task. In contrast, children performed somewhat less well on intention and consequence questions in the relevant moral vignette (true belief, bad intent) than on the same questions in the standard moral transgression task. Two possible factors may have generated this difference. First, our vignettes were more informationally more complex than the standard task and, second, the outcome was not as salient in our vignettes (an undesirable object hidden in a container) as in the standard task (a character falling down and hurting his or her knee). 
We turn now to the central findings for the moral vignettes under different conditions. As expected, in true belief trials children generally performed well in responding to intent and consequence questions: They rated agents as worse intentioned and more deserving of punishment in bad intent conditions compared to good intent conditions. Moreover, this pattern was found for false belief failers as well as false belief passers, which is of course not surprising as false belief understanding was not required for success on the true belief trials.

In contrast, on false belief trials performance on the intent questions interacted with false belief status. Confirming and extending previous research on good intent (Killen et al., 2011), we found that the acquisition of false belief understanding does indeed change children's patterns of moral evaluations in a false belief context, and does so for both good and bad intent.

Not surprisingly, false belief failers performed poorly on intent questions -- assigning intent accurately depended on understanding that agents had false beliefs. More interestingly, a developmental lag emerged between false belief understanding and integrating that understanding with moral evaluations. Five-year-old false belief passers rated agents as more positively intentioned in good intent trials (even though the outcome was bad) than in bad intent trials (even though the outcome was good). In contrast, 4-year-old false belief passers were generally unable to integrate their belief understanding with their moral evaluations, performing no better on intention questions than false belief failers. Unlike false belief failers, this group correctly predicted that the agent had a mistaken belief about the container he/she shared with the recipient, but were unable to use this information in assessing the agent's intention. This finding is in accord with previous work (de Rosnay et al., 2004) in which children were not able to use false belief information to make false belief-influenced emotional predictions in a similarly-aged sample. It is possible, however, that by reducing the 
information processing demands in the moral vignettes, 4-year-old passers may be better able to use their belief knowledge to make moral judgments. We explore this possibility in Study 2.

As noted earlier, children performed quite well on the agent consequence questions in true belief trials: They assigned greater punishment to agents with ill intent who generated negative outcomes for recipients than to those with good intent who generated positive outcomes for recipients. In contrast, on false belief trials, even 5-year-old false belief passers performed no better than chance when assigning a consequence to the agent across levels of intent. As hypothesized, a further developmental lag emerged between incorporating belief understanding in intent judgments and doing so in consequence judgments. What is not clear from the present study is how long that lag lasts since even the oldest children struggled with consequence questions. We begin to address this issue in Study 2.

\section{Study Two}

The purpose of study two was four-fold. First, we aimed to investigate whether the developmental lags found in Study 1 would replicate when the processing demands of the task and the length of the testing session were reduced. We simplified the design of the moral vignettes in two ways. We used only animals (and not food items) so that children would only have to direct their attention to the animals of interest rather than a third animal entering and moving the food items from one box to another. In addition, we used only a single box such that children would not have to track the movements of two animals across two separate boxes.

To shorten the testing session, we also dispensed with the standard belief and moral tasks used in Study 1, and we reduced the number of test questions for each moral vignette by combining the agent evaluation and agent intention questions into a single question and 
dropping the friend question. In addition, because children performed so well on true belief trials in Study 1, we only administered false belief trials in Study 2.

Second, to gain deeper insights into children's thinking about assigning consequences, we asked open-ended questions probing the reasons behind their judgment of deserved reward or punishment. In this way, we hoped to uncover underlying moral principles that might guide children's reasoning (Cushman et al., 2006). Third, because even 5-year-olds in our first study did not appropriately assign reward/punishment on false belief trials (and neither did some of the 7-year-olds in Killen et al., 2011), we included an adult sample for comparison purposes to determine a developmental endpoint for consequence reasoning. Finally, because some of the cell sizes were on the low side for our false belief groups, we increased the sample size for Study 2 so that the central tests of our hypotheses would be better-powered.

Following from the findings of Study 1, we hypothesized that children who did not show false belief understanding, would not be able to appropriately attribute agent intentions, but would instead tend to answer intention questions based on outcome, and would do so more often than false belief passers. We also hypothesized a developmental lag for use of false belief information in response to intention questions, such that adults and 5-year-old false belief passers would make more appropriate intent-based judgments than 4-year-old passers. We also hypothesized that 4-year-old passers would have more difficulty assigning punishment/reward than 5-year-old passers and adults. Finally, we hypothesized that punishment/reward judgments would again be harder than intent judgments, particularly for 4- and 5-year-olds. Our hypotheses and analysis plan were preregistered prior to analyzing the data on the OSF.

\section{Method}




\section{Participants}

One-hundred and nine children participated, 63 4-year-olds (34 girls; $M_{\text {age }}=53.80$ months, $S D=3.56)$ and 465 -year-olds $\left(21\right.$ girls; $M_{\text {age }}=65.70$ months, $\left.S D=3.33\right)$. An additional 5 children were tested but excluded from analyses due to experimenter error (3), inattention (1), and family interference (1). The sample was representative of the population from which it was drawn. Eighty-six children were White, 5 reported being two or more races/ethnicity, 3 were Asian, 2 were Hispanic, Latino, or Spanish, 2 were Middle Eastern, and 1 was Native American/Native Alaskan. Ten parents did not report their child's race or ethnicity. Seventy-five percent of families reported making at least $\$ 40,000$ a year.

Eighty-nine children participated in the lab and were recruited from a participant database at a large research university in the Western United States. Parents were provided compensation in the form of $\$ 10$, and children received a small toy. A further 25 children participated in a quiet space at a local children's museum -- parents of these children did not receive compensation, but children received stickers.

Forty-two adults ( $69 \%$ female, $M_{a g e}=19.71$ years, $S D=2.50$ years) from an undergraduate participant database also participated. The majority (26) reported being White, 8 were Asian or Asian-American, 2 were Black, and 6 reported being of two or more race/ethnicities or other. Participants received compensation in the form of class credit.

\section{Design}

Participants were randomly assigned to one of two intent conditions (good or bad) in which they responded to two morally-relevant belief vignettes. An a priori analysis indicated at least $30 \mathrm{FB}$ passers would be required in each age group (4-year-olds, 5-year-olds, and adults) for the study to be adequately powered. Because we anticipated that many children would fail the false belief (FB) task, we oversampled in order to achieve our targeted 
numbers of FB passers. Ultimately, we recruited 63 4-year-olds (30 FB passers), 46

5-year-olds (31 FB passers), and 42 adults (40 FB passers).

\section{Procedure}

Children who participated in the lab were tested individually in a single 5-10 min videotaped session. Children who participated at the local children's museum were tested in a quiet space in a single session. Adults completed all tasks in the lab on an iPad.

\section{Measures}

Morally-relevant belief vignettes. Similar to Study 1, participants in each age group were randomly assigned to the two intent conditions until the required sample size of 30 was achieved: for children, good intent $\left(N=46,28\right.$ girls, $M_{\text {age }}=57.70$ months $)$ or bad intent $(N=$ 59, 26 girls, $M_{\text {age }}=60$ months $)$ and, for adults, good intent $\left(N=22,14\right.$ women, $M_{\text {age }}=19.86$ years) or bad intent $\left(N=20,15\right.$ women, $M_{\text {age }}=19.75$ years $)$. Each participant heard two morally-relevant belief vignettes(one with a boy agent and one with a girl agent) in which the agent held a FB, with vignette order counterbalanced across conditions. As in Study 1, good intent stories involved an agent who wanted to make a friend happy by sharing a desirable animal (kitten/butterfly). Because of a FB the agent ends up sharing an undesirable animal (skunk/spider). In the bad intent stories, the agent wanted to make their friend upset, but because of a FB ended up sharing a desirable animal. Unlike Study 1, the vignettes involved only one box as follows. The agent first opened the box, stating what animal was in it and whether it would make their friend happy or sad. The next animal then appeared on the scene with the agent stating whether it would make the friend happy or sad. At this point the agent left, after which the animal in the box jumped out and also left. The other animal then jumped in the box. The agent then returned and, when their friend arrived, they gave them the box that now contained the unintended animal. 
Following each vignette, participants responded to six questions in a fixed order: (1) intention evaluation ("When [AGENT] handed [RECIPIENT] the box, was [AGENT] trying to be nice, mean, or just okay?"; (2) agent belief; (3) reality check; (4) consequence evaluation; (5) open ended consequence explanation ("Why should [AGENT] get [assigned consequence)?]; and (6) recipient emotion attribution. Response choices were offered in a fixed order. The same three comprehension questions from Study 1 were again included.

The agent belief, reality check, consequence evaluation, and recipient emotion attribution questions were identical to those in Study 1 and were coded as in that study. The new intent evaluation question was coded on the same scale as the agent intention and evaluation questions from Study 1.

Participants' responses to the open-ended punishment/reward questions were coded for three purposes: (1) content of the justification, (2) correctness of the justification, and (3) whether the justification matched the assigned punishment/reward. The content of the justification was coded as referencing: Mental state, such as, intention, knowledge, or belief (e.g., "she didn't know the spider got in the box", "trying to make her friend happy"); Outcome (e.g., "there's a butterfly in the box"); or Undifferentiated/Uninterpretable (e.g., "I don't know”, or "just because"). The justification was also coded for correctness (i.e., judging whether it matched facts from the story): Correct (e.g., "she was trying to be nice" in the good intent condition), Incorrect (e.g., "she was trying to be mean" in the good intent condition), or Undifferentiated/Uninterpretable. Lastly, the justification was coded for whether it matched the assigned punishment/reward (e.g., assign a reward to an agent because "she was trying to do something nice") or Incorrect (e.g., assign a reward to the agent even though they state that "She was being mean"). In most cases assigning nothing, (no treat or reward) was coded as a match because that would be considered acceptable in both scenarios. 
If the initial justification was coded as undifferentiated, it remained undifferentiated in this coding. Two independent coders attained high inter-rater reliability (Cohen's $K=.82$ ), with 90.3\% agreement across 792 observations. All discrepancies were resolved in discussion between the two coders and the first author.

Adults answered the same questions as children. The only difference was that adults were asked the three comprehension questions at the conclusion of each vignette because we were not concerned about memory demands for them, whereas children were asked during each vignette.

\section{Results}

No main effects of gender, story order (girl vs boy story first), or experimenter were found. Therefore, these factors were collapsed in subsequent analyses. As in Study 1, we used the recipient emotion prediction question as a comprehension check. The majority of children and adults appropriately judged that the recipient would feel sad in the good intent condition $(M=-.88, S D=.42$, and $M=-.96, S D=.21$, respectively $)$ and happy in the bad intent condition $(M=.69, S D=.67$, and $M=.60, S D=.68$, respectively $)$.

\section{Agent Belief}

A 2 (intent: good vs. bad) x 2 (age: 4- vs. 5-year-olds) ANOVA was conducted in response to the agent belief question. Adults were not included in this analysis because they were essentially at ceiling in both conditions: good intent $(M=1, S D=0)$; bad intent condition $(M=.98, S D=0.11)$. A main effect of age revealed 5-year-olds $(M=0.74, S D=$ $0.41)$ ascribing the appropriate belief more often than 4-year-olds $(M=0.58, S D=0.46)$, $F(1,105)=4.41, p=.04, \eta_{p}^{2}=.03$. The main effect of intent and the interaction were not significant. As in Study 1, the sample was then divided into groups as a function of belief understanding: participants who failed one or both belief questions $\left(N=50,48\right.$ children, $M_{\text {age }}$ 
$=57.80$ months, $S D=6.76$ months, and 2 adults $), 4$-year-olds who passed both $\left(N=30, M_{\text {age }}\right.$ $=53.60$ months, $S D=3.19)$, 5-year-olds who passed both $\left(N=31, M_{\text {age }}=65.6\right.$ months, $S D=$ 3.39), and adults who passed both $(N=40)$.

\section{Agent Intention}

Figure 3 (panel A) depicts performance on the intention question. Adults were at ceiling: They reported that the agent in the bad intent condition was trying to be mean and the agent in the good intent condition was trying to be nice. For children, a 3 (FB group) x 2 (intent) factorial ANOVA revealed a main effect of intent, $F(1,102)=28.95, p<.001, \eta_{p}^{2}=$ .22 , but not a main effect of FB group, $F(1,102)=1.67, p=0.19$. Overall, children appropriately rated agents in the good intent condition $(M=0.32, S D=.70)$ as significantly better intentioned than those in the bad intent condition $(M=-0.34, S D=.68)$. However, this main effect was qualified by a FB group by intent interaction, $F(2,102)=9.13, p=.004, \eta_{p}^{2}$ $=.10$. Follow up simple effects comparisons of intent for each FB group revealed that FB failers did not significantly differentiate intention ratings across the good and bad intent conditions, $p=.40$. In contrast, 4- and 5-year-old passers appropriately rated agents in the good intent condition as significantly better intentioned than agents in the bad intent condition, $t s(29$ and 30$)=-3.61$ and $-5.15, p s=.006$ and $<.001$, respectively. Further, 4and 5-year-old passers performed significantly better than chance in all conditions $(p s<.02)$, except for 4-year-olds in the good intent condition who were only marginally different from chance, $t(13)=1.86, p=.09$.

\section{Agent Consequence}

Figure 3 (Panel B) depicts performance on the agent consequence question. A 4 (group: FB failers, 4-year-old passers, 5-year-old passers, and adult passers) x 2 (intent: good vs. bad) factorial ANOVA revealed a main effect of FB group, $F(3,142)=5.92, p<.001$, 
$\eta_{p}{ }^{2}=.11$, and a significant interaction between FB group and condition, $F(3,142)=5.37, p=$ $002, \eta_{p}{ }^{2}=.10$. The main effect of intent was not significant, $F(1,142)=2.19, p>.05, \eta_{p}{ }^{2}=$ .02. Follow up simple effects comparisons revealed that only adults appropriately rated agents in bad intent conditions as deserving more punishment than those in good intent conditions, $t(39)=-3.92, p=.003$. No other FB group distinguished punishment ratings for agents in the good versus bad intent conditions $(p s>.05)$.

We conducted the same follow-up analysis as in Study 1, to examine whether FB groups differed in whether their consequence ratings were correct or incorrect. Adults were excluded because their ratings in both conditions were at ceiling. For children, a 3 (FB group) x 2 (intent) ANOVA on correct consequence ratings (0-2) did not reveal any main effects or an interaction between FB group and intent condition ( $p s>.05)$. FB failers $(52 \%$ correct), 4-year-old passers (53\% correct), and 5-year-old passers ( $48 \%$ correct) had similar difficulty assigning consequences in the FB context, with no group performing better than chance, $t \mathrm{~s}(47,28,30)=-0.17,0.90,-0.31, p \mathrm{~s}>.37$.

\section{Justification for Punishment or Reward}

Finally, we examined the proportion of justifications for punishment/reward broken down by FB group for the content of the justification, correctness of the justification, and whether the justification matched the assigned punishment/reward. Because 4- and 5-year-olds FB passers revealed essentially the same pattern of justifications across the three codes, we collapsed them for this analysis. For children, a large portion of justifications were coded as undifferentiated/uninterpretable (43\% vs. 10\% for adults). Of the justifications that were interpretable, the majority for all FB groups matched the assigned punishment/reward: 92\% FB failers, 97\% Child passers, and 100\% Adult passers. 
With respect to the content of the justifications, mental states were referred to more often than outcomes, and that was true even for FB failers (see Table 1). In a further analysis, however, we examined whether the mental state reference was consistent with the story. Even though FB failers referenced mental states more than outcomes, they often did so incorrectly. Only $42 \%$ of FB failers who referenced a mental state did so correctly, versus $85 \%$ for child FB passers and $97 \%$ for adult passers. The other $58 \%$ of FB failers referenced mental states that were inconsistent with the vignettes (e.g., stating that "he wanted to give his friend something nice" in the bad intent condition or "because she did something mean" in the good intent condition).

\section{Discussion}

In this study we examined whether the developmental differences found in the first study would persist when information processing demands were reduced. In contrast to Study 1, both 4- and 5-year-old false belief passers appropriately rated agents in the good intent condition as significantly better intentioned than agents in the bad intent condition. In the first study only 5-year-olds passers had made this distinction. It appears that reducing the task processing demands may have helped younger children focus on the relevant information needed to make accurate moral judgments.

We had also hypothesized that 4-year-old passers would have more difficulty assigning appropriate consequences than 5-year-old passers. However, we again found that children of all ages, regardless of false belief understanding, had difficulty doing so. Despite the reduced processing demands, children did not significantly differentiate consequence ratings for agents in the good versus bad intent conditions.

In addition, we hypothesized that consequence judgments would be harder than intent judgments for children. As in Study 1, this hypothesis was confirmed: 4- and 5-year-old false 
belief passers were often able to properly assign agent intent, but generally did not make appropriate use of this information when assigning a punishment/reward. With respect to consequence justifications, we found that all groups tended to reference mental states more often than outcomes. This initial finding was surprising because previous research suggests that younger children, and especially those without false belief understanding, often focus more on outcomes when assigning consequences (Cushman et al., 2013; Zelazo et al., 1996). However, when examining whether participants' references to mental states were accurate, children without false belief understanding often incorrectly referenced agents' intentions by aligning those intentions with their mischaracterizations of agents' beliefs. For example, in the good intent condition, they appeared to think that the agent knew there was an unpleasant object in the container, and therefore they thought he or she must have a bad intention. As a result, they recommended punishment for the agent. In that sense, these children were perhaps still outcome-focused -- they assigned intentions to the agent in their justifications that matched the outcome of the vignette.

Lastly, we found that 4- and 5-year-old false belief passers' moral judgments differed from those of adults in several respects. Both children and adults significantly distinguished good intentions from bad intentions. However, whereas adults were at ceiling in doing so, children were much more variable. Moreover, while children did not significantly distinguish good versus bad intent in their consequence judgments, adults had little difficulty in doing so.

\section{General Discussion}

Although the influence of intent understanding on children's moral development has been long studied (e.g., Piaget, 1932), very little research has examined the influence of belief understanding on that development. In two studies we presented children with morally-relevant belief vignettes to examine the extent to which they incorporate both intent 
and belief information in their moral judgements. By sampling within the narrow developmental window during which false belief understanding is acquired, we found important developmental changes in children's ability to integrate mental state understanding with moral reasoning.

In Study 1, when agents held true beliefs, 4- and 5-year-olds appropriately shifted their moral judgments according to whether the agent had a good or bad intention, and children did so irrespective of whether they demonstrated understanding of false beliefs. Children appropriately rated agents sharing pleasant containers as positively intentioned and deserving of reward, while rating agents sharing unpleasant containers as negatively intentioned and deserving of punishment. These findings are important because they show that children of this age have a basic understanding of the role of intent in determining both the moral status of agents and whether or not those agents deserve punishment or reward.

Yet, in the otherwise similar false belief conditions, the pattern was quite different. In those conditions, we anticipated that the intersection of intent and false belief would lead to differential response patterns across the sample concordant with the attainment of false belief understanding. We predicted that children without false belief understanding would perform poorly, responding in terms of outcome when assessing intent and consequences. For children with false belief understanding, we had two contrasting hypotheses. If they immediately integrate such understanding with moral reasoning, they should use this information to correctly infer agents' intentions. In contrast, if there is an integration delay, the younger of these children might perform poorly, again defaulting to outcome-based or other incorrect responses. Our findings were consistent with the latter hypothesis: 5-year-old false belief passers, but not 4-year-old passers, appropriately shifted their evaluations as a function of intent in cases where agents held false beliefs. Further, as hypothesized, we found support for 
a developmental lag between appropriately assigning intent and appropriately assigning consequences. Even 5-year-old false belief passers did not distinguish ratings of punishment or reward as a function of intent in false belief contexts (whereas they had done so successfully in true belief contexts).

In Study 2, we attempted to replicate the developmental lag in conditions in which the task processing demands were reduced. We did so by using only animals (and not food items) so that children would only have to direct their attention to the animals of interest rather than a third animal entering and moving the food items from one box to another. Further, we used only a single box such that children would not have to track the movements of two animals across two separate boxes. We also added an adult comparison group to establish a developmental endpoint, hypothesizing that 4-year-old false belief passers would have more difficulty assigning consequences than 5-year-old passers and adults. Finally, we believed that consequence judgments would again be more difficult than intent judgments, particularly for 4- and 5-year-olds.

We found that when processing demands were reduced, the developmental lag for intent judgments was no longer evident: 4-year-old, as well as 5-year-old false belief passers, now performed better than chance in attributing agents' intentions. Nonetheless, children of both ages continued to have difficulty making appropriate punishment/reward judgments, indicative of a developmental lag between assigning moral intent and assigning consequences. In contrast, adults showed no such difficulty, performing near ceiling on both intent and consequence questions (when the latter were scored as correct/incorrect).

Our findings are both similar to and different from those of Killen et al. (2011) who also found that false belief understanding is related to moral judgment. Specifically, they found that only the oldest children (7-8 year-olds) attributed good intentions to an accidental 
transgressor in a false belief context in which a good intention generated moral violation. We extended Killen et al 's approach by also including scenarios in which an agent had a negative intention, but due to a false belief ending up making a friend happy. Like Killen et al., we found that false belief understanding was linked to moral judgment. However, unlike Killen et al., our children made the link at an earlier age, with many 5-year-olds showing an understanding of moral intentions in the false belief context in Study 1, and many 4-year-olds doing so in Study 2.

Further, children in Killen et al. (2011) deemed punishing the accidental transgressor as less acceptable with age, whereas children in our studies did not make a clear distinction in whether to punish or reward agents in either the positive or negative intent condition. The findings across their research and ours are difficult to compare, however, because the question formats differ and because their study included older children. That said, their 5-year-olds appeared to have had similar difficulty to children of that age in our sample.

Why is it that the developmental lag in integrating false belief understanding with intent judgments largely disappeared in study 2 ? In Study 2, we shortened the testing session, simplified the procedure, and only had children respond to false belief vignettes. As aforementioned, reducing information processing demands may have allowed 4-year-olds to apply their false belief understanding to their judgment of intent.. Nonetheless, it is worth noting that, even though performance improved in Study 2, not all 4- and 5-year-old false belief passers correctly inferred the intent. In contrast, adults were at ceiling, indicating that a lag still exists before children reach adult levels.

Our findings with respect to the difficulty children have in assigning reward and punishment appropriately parallel some findings from Cushman et al. (2013). They found that while children can make appropriate moral wrongness judgments based on intent, their 
punishment judgments are based more on outcome. Specifically, in Cushman et al's research children were asked to make wrongness judgments (presumably based on intent) and punishment judgments for cases of accidental harm (benign intent, bad outcome) versus attempted harm (negative intent, neutral outcome). Children aged 5 and older assigned higher levels of punishment than wrongness in the case of accidental harm, and, conversely, higher levels of wrongness than punishment in the case of attempted harm. In contrast, 4-year-olds did not clearly distinguish punishment and wrongness in either case. Thus, as in our studies, appropriately incorporating consequences on the basis of intent was difficult for children.

\section{Future Directions}

Our findings might be followed up in a variety of ways. First, unlike adults, even the oldest children in our sample failed to assign consequences differentially as a function of intent in the false belief context. As a result, we do not know when in development children reach adult levels of understanding. Hence, it will be important to include older age groups in future work.

Second, it is possible that our scenarios were perplexing to some children given that no explicit reason was provided as to why agents would want to make their friend happy or sad. Although making a friend happy is perhaps something of a default, it may be harder to take in why someone would want to make a friend sad without a specific reason. While children did not appear to be confused about this fact in the true belief context, it may have proved harder to integrate that information when a false belief was in the mix. Clarifying the reasons behind agents' actions may therefore be an important addition in future work.

Third, asking children whether they want to praise or blame an agent, may reveal additional information regarding moral judgments. It is possible that praise and blame would be more closely linked to intention (and belief) than would reward and punishment. While we 
may hesitate to give rewards when outcomes are inadvertently bad, we may feel more free to offer praise for good intentions in those cases; conversely, while we may not punish when good outcomes fortuitously occur, we may nonetheless be quite willing to blame ill-intentioned agents in such cases. In that respect, praise and blame may represent something of a way station on children's road to incorporating mental states into judgments of punishment and reward.

Finally, another aspect of theory of mind that is important for moral development is emotion understanding and empathy (Hoffman, 2000). For example, Ball et al. (2017) found that greater empathy in young children was associated with judgments of severity/wrongness of moral transgressions but not social conventional transgressions. Interestingly, they also found that empathy was related to criterion judgments, such as, in deciding whether it would be okay to act immorally if an authority figure said it was okay, or if there were no social or cultural rules about the act; yet, this was only true for children with lower ToM. The researchers suggest that children with lower ToM may rely more on affective information; for example, they empathize with the impact of the negative outcome on the victim when judging immoral acts, and may not necessarily need ToM in order to make these judgments. In scenarios like the ones we presented to children, it would thus be interesting to assess the extent to which empathy influences judgments of intent and punishability.

\section{Conclusion}

In sum, we found revealing interactions between false belief understanding and moral judgment. Although children are able to reason about intent (good and bad) in contexts in which an agent holds a true belief, it is only as children attain false belief understanding that they appropriately reason about intent in contexts in which an agent holds a false belief. Moreover, moral reasoning develops beyond the preschool years, as even 5-year-olds did not 
appropriately assign punishment/reward as a function of intent, and their understanding of moral intent itself lagged behind that of adults. Integrating theory of mind and moral judgment is clearly a complex developmental achievement that is vital for positive social relationships. That achievement is not, however, attained in a single step. 


\section{References}

Apperly, I. A., Warren, F., Andrews, B. J., Grant, J., \& Todd, S. (2011). Developmental continuity in theory of mind: Speed and accuracy of belief-desire reasoning in children and adults. Child development, 82(5), 1691-1703. https://doi.org/10.1111/j.1467-8624.2011.01635.x

Astington, J. W. (2003). Sometimes necessary, never sufficient: False-belief understanding and social competence. In B. Repacholi \& V. Slaughter (Eds.), Individual differences in theory of mind: Implications for typical and atypical development (pp. 13-38). New York: Psychology Press.

Baird, J. A., \& Astington, J. W. (2005). The development of the intention concept: From the observable world to the unobservable mind. The new unconscious, 256-276. http://dx.doi.org/10.1093/acprof:oso/9780195307696.003.0011

Ball, C. L., Smetana, J. G. and Sturge-Apple, M. L. (2017), Following My Head and My Heart: Integrating Preschoolers' Empathy, Theory of Mind, and Moral Judgments. Child Development, 88, 597-611.https://doi.org/10.1111/cdev.12605

Baron-Cohen, S., Leslie, A. M., \& Frith, U. (1985). Does the autistic child have a "theory of mind"?. Cognition, 21(1), 37-46. https://doi.org/10.1016/0010-0277(85)90022-8

Boseovski, J. J. (2010). Evidence for "rose-colored glasses": An examination of the positivity bias in young children's personality judgments. Child Development Perspectives, 4(3), 212-218. https://doi.org/10.1111/j.1750-8606.2010.00149.x

Cowell, J. M., Lee, K., Malcolm-Smith, S., Selcuk, B., Zhou, X., \& Decety, J. (2017). The development of generosity and moral cognition across five cultures. Developmental Science, 20(4), e12403. https://doi.org/10.1111/desc.12403 
Cushman, F., Sheketoff, R.W., Wharton, S., \& Carey, S. (2013). The development of intent-based moral judgment. Cognition, 127, 6-21. https://doi.org/10.1016/j.cognition.2012.11.008

Cushman, F., Young, L., \& Hauser, M. (2006). The role of conscious reasoning and intuition in moral judgment: Testing three principles of harm. Psychological science, 17(12), 1082-1089. https://doi.org/10.1111/j.1467-9280.2006.01834.x

De Rosnay, M., Pons, F., Harris, P. L., \& Morrell, J. M. (2004). A lag between understanding false belief and emotion attribution in young children: Relationships with linguistic ability and mothers' mental-state language. British Journal of Developmental Psychology, 22(2), 197-218. https://doi.org/10.1348/026151004323044573

Hoffman, M. (2000). Empathy and moral development. Implications for justice and caring. Cambridge, UK: Cambridge University Press. https://doi.org/10.1017/CBO9780511805851

Killen, M., Mulvey, K. L., Richardson, C., Jampol, N., \& Woodward, A. (2011). The accidental transgressor: Morally-relevant theory of mind. Cognition, 119(2), 197-215. https://doi.org/10.1016/j.cognition.2011.01.006

Knobe, J. (2003). Intentional action in folk psychology: An experimental investigation. Philosophical psychology, 16(2), 309-324. https://doi.org/10.1080/09515080307771

Lagattuta, K., \& Weller, D. (2014). Interrelations between theory of mind and morality. Handbook of moral development, 385-407. https://doi.org/10.4324/9780203581957

Lane, J., Wellman, H., Olson, S., LaBounty, J., \& Kerr, D. (2010). Theory of mind and emotion understanding predict moral development in early childhood. British Journal of Developmental Psychology, 28(4), 871-889.

https://doi.org/10.1348/026151009X483056 
Leslie, A. M. (1988). Some implications of pretense for mechanisms underlying the child's theory of mind. In J. W. Astington, P. L. Harris, \& D. R. Olson (Eds.), Developing theories of mind (pp. 19-46). New York, NY, US: Cambridge University Press.

Leslie, A. M., German, T. P., \& Polizzi, P. (2005). Belief-desire reasoning as a process of selection. Cognitive Psychology, 50(1), 45-85. https://doi.org/10.1016/j.cogpsych.2004.06.002

Leslie, A. M., Knobe, J., \& Cohen, A. (2006). Acting Intentionally and the Side-Effect Effect: Theory of Mind and Moral Judgment. Psychological Science, 17(5), 421-427. https://doi.org/10.1111/j.1467-9280.2006.01722.x

Piaget, J. (1965/1932). The moral judgment of the child. New York: Free Press.

Premack, D., \& Woodruff, G. (1978). Does the chimpanzee have a theory of mind?. Behavioral and brain sciences, 1(4), 515-526. https://doi.org/10.1017/S0140525X00076512

Smetana, J. G. (2006). Social domain theory: Consistencies and variations in children's moral and social judgments. In M. Killen \& J. G. Smetana (Eds.), Handbook of moral development (pp. 119-153). Mahwah, NJ: Erlbaum.

Smetana, J., Jambon, M., Conry-Murray, C., \& Sturge-Apple, M. (2012). Reciprocal associations between young children's developing moral judgments and theory of mind. Developmental Psychology, 48(4), 1144-1155. https://doi.org/10.1037/a0025891

Wainryb, C., \& Ford, S. (1998). Young children's evaluations of acts based on beliefs different from their own. Merrill-Palmer Quarterly, 484-503.

Wellman, H. M. (2020). Reading Minds: How Childhood Teaches Us to Understand People. Oxford University Press, USA. 
Wimmer, H., Gruber, S., \& Perner, J. (1984). Young children's conception of lying: Lexical realism—moral subjectivism. Journal of Experimental Child Psychology, 37(1), 1-30. https://doi.org/10.1016/0022-0965(84)90055-9

Yuill, N., \& Perner, J. (1988). Intentionality and knowledge in children's judgments of actor's responsibility and recipient's emotional reaction. Developmental psychology, 24(3), 358. https://doi.org/10.1037/0012-1649.24.3.358

Zelazo, P. D., Helwig, C. C., \& Lau, A. (1996). Intention, Act, and Outcome in Behavioral Prediction and Moral Judgment. Child Development, 67(5), 2478-2492. https://doi.org/10.2307/1131635 
Table 1.

Proportion of Justification Types for Punishment/Reward by False Belief Group

\begin{tabular}{llccc}
\hline $\begin{array}{l}\text { False Belief } \\
\text { Group }\end{array}$ & $\begin{array}{l}\text { Number of } \\
\text { Justifications }\end{array}$ & & \multicolumn{3}{c}{ Content of Justification } \\
\hline & & Mental State & Outcome & Undifferentiated \\
\cline { 2 - 5 } FB Failers & 100 & .39 & .15 & .46 \\
Child Passers & 122 & .39 & .22 & .39 \\
Adult Passers & 80 & .88 & 0 & .12 \\
\hline
\end{tabular}



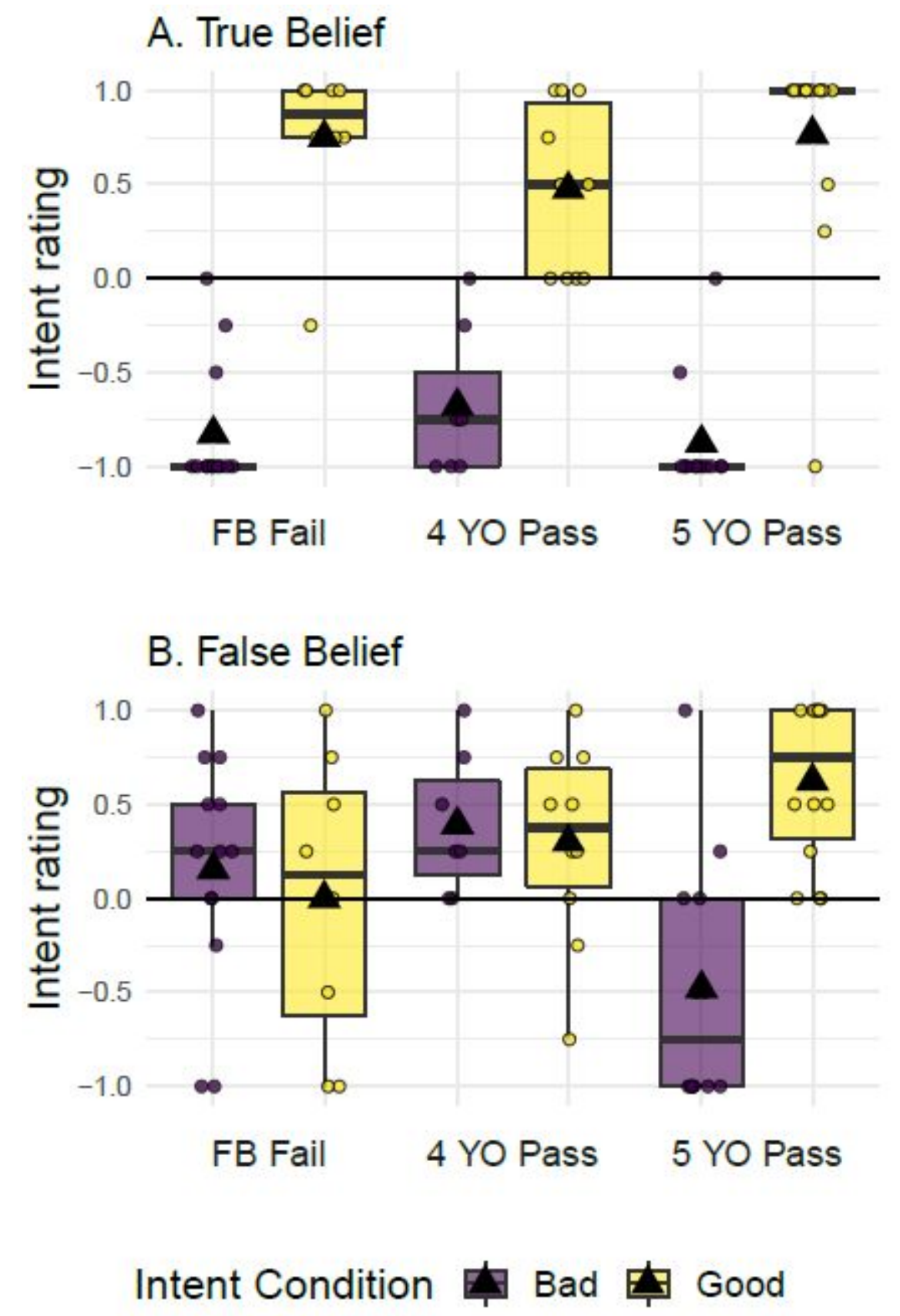

Figure 1. Boxplot with mean (indicated by a black triangle), and individual responses for the agent intent composite for each of the three belief groups for the true belief condition (top) and false belief condition (bottom). 

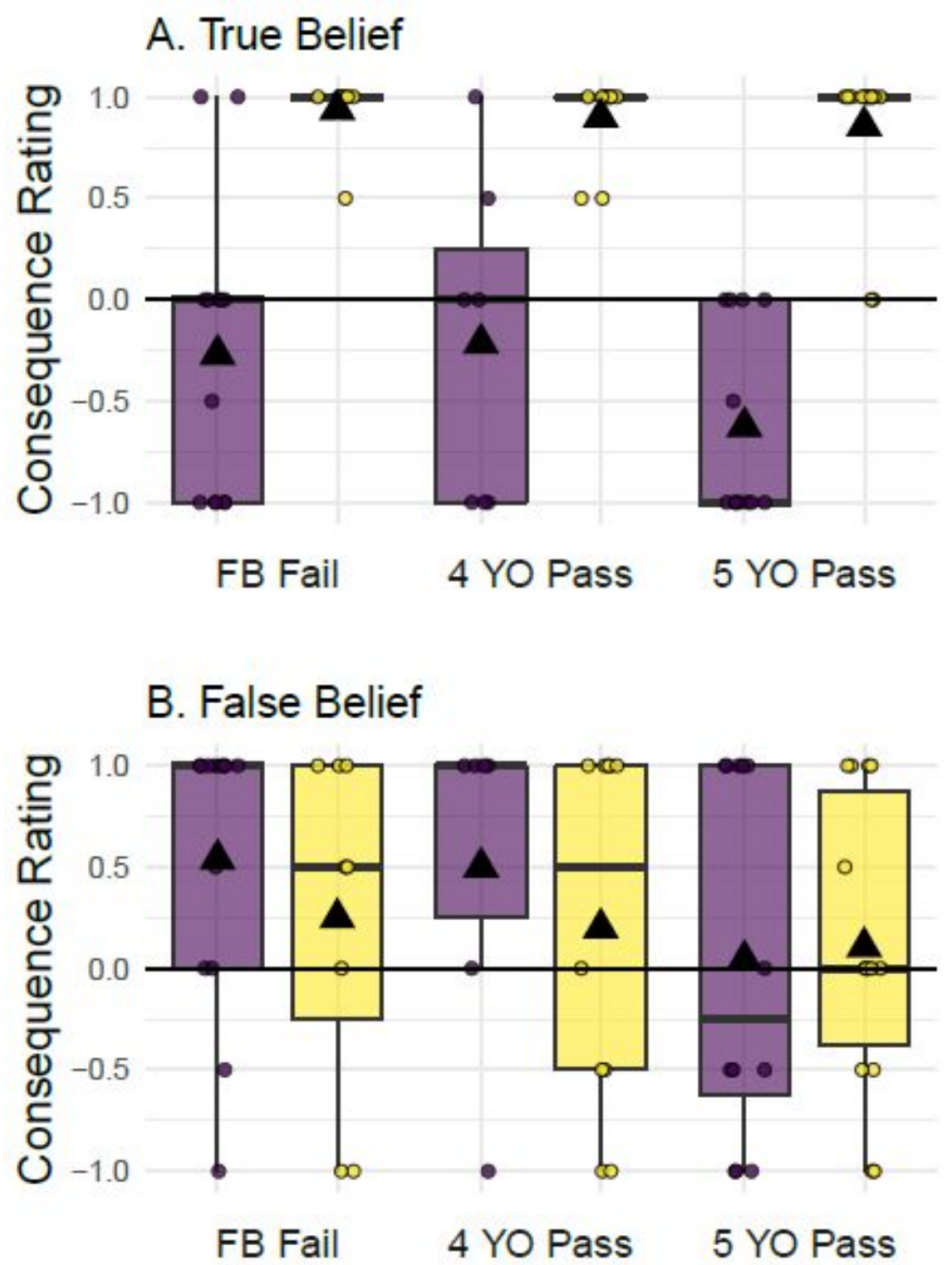

Intent Condition Bad Good

Figure 2. Boxplot with mean (indicated by a black triangle), and individual responses to the consequence question for each of the three belief groups for the true belief condition (top) and false belief condition (bottom). 

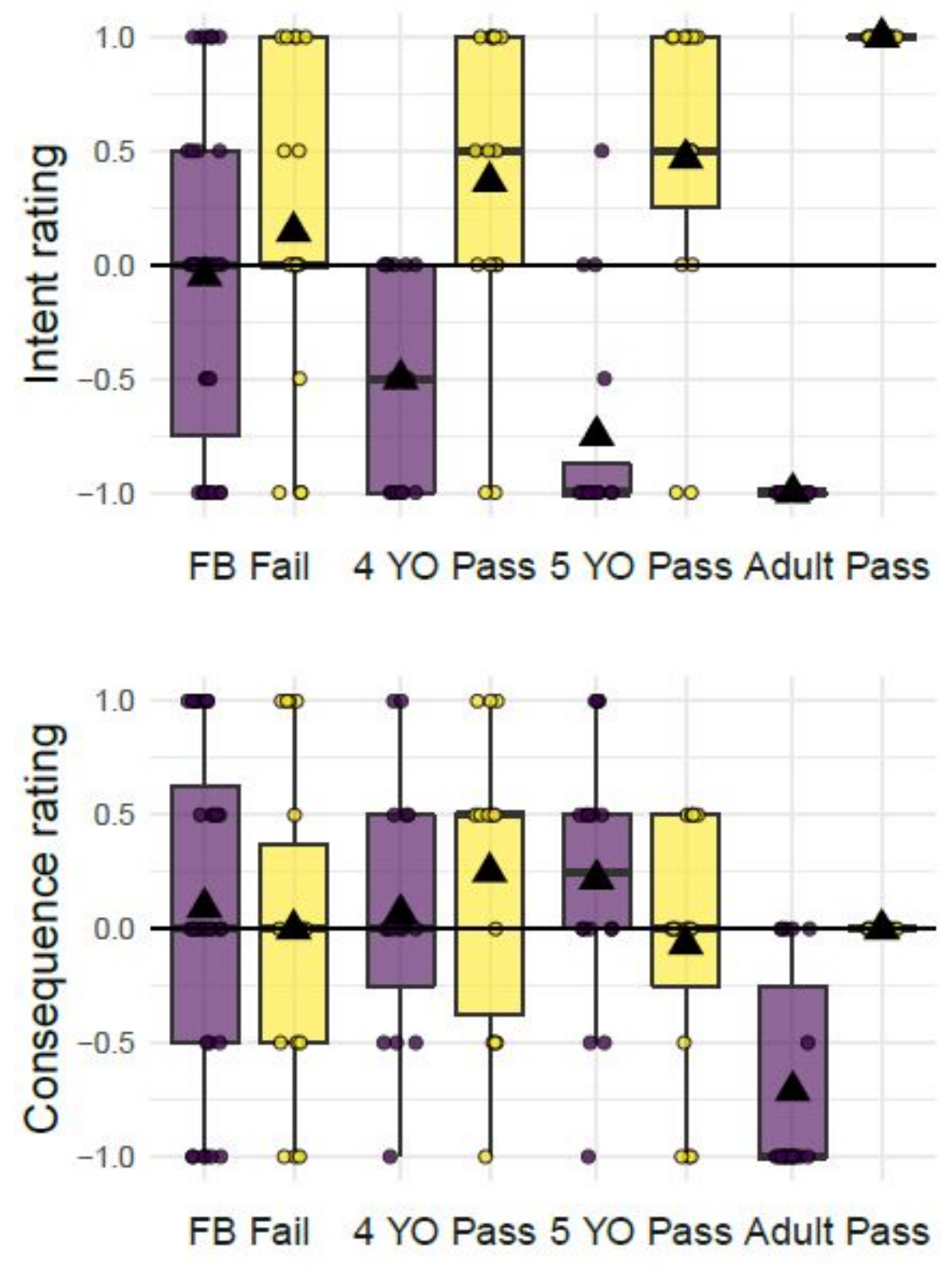

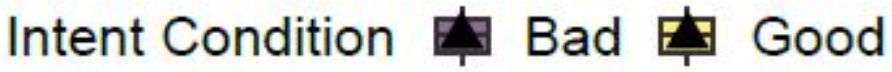

Figure 3. Boxplot with mean (indicated by a black triangle), and individual responses to the intent (top panel) and consequence questions for each of the belief groups. 